\title{
DE LA INSTRUMENTACIÓN ELECTRÓNICA DEL AUTOMÓVIL
}

\section{IN AUTOMOTIVE ELECTRONICS INSTRUMENTATION}

\section{AUTORES}

Arturo Pérez París: Alumno de la Escuela Politécnica de la Universidad de Alcalá. arturo.perez@aol.com

\section{CURRÍCULUM VITAE}

Alumno de la Escuela Politécnica de la Universidad de Alcalá de Henares (España). Ingeniero de Soporte Técnico en Kone Elevadores

\section{RESUMEN}

Hace unos meses publicamos una pequeña introducción a la electrónica básica de nuestros coches. En aquella ocasión, ya anuncié que se trataría del primero, dentro una pequeña serie artículos sobre el tema. Pues bien, como el que avisa no es traidor, les presento esta segunda parte, y ya les adelanto que se preparará en breve una tercera. Espero con esto no aburrirles en exceso y que puedan sacar algún provecho en las futuras ñapas automovilísticas que tengan que realizar. Sin más dilación comencemos con los circuitos para la medida de la temperatura del agua del radiador o del aceite del motor.

\section{PALABRAS CLAVE}

Automóvil - Instrumentación electrónica - Mecánica 


\section{ABSTRACT}

A few months ago we published a short introduction to basic electronics of our cars. At that time, I announced that it would be the first in a small series of articles on the subject. Well, as that Forewarned is forearmed, I present this second part, and as they advance to be prepared shortly third. I hope this does not bore you too much and can get some profit on future groundwater automakers have to make. Without further delay circuits to start with the measurement of the water temperature, the radiator or engine oil.

\section{KEY WORDS}

Automotive - Electronic - Mechanical

Hace unos meses publicamos una pequeña introducción a la electrónica básica de nuestros coches. En aquella ocasión, ya anuncié que se trataría del primero, dentro una pequeña serie artículos sobre el tema. Pues bien, como el que avisa no es traidor, les presento esta segunda parte, y ya les adelanto que se preparará en breve una tercera. Espero con esto no aburrirles en exceso y que puedan sacar algún provecho en las futuras "ñapas automovilísticas" que tengan que realizar. Sin más dilación comencemos con los circuitos para la medida de la temperatura del agua del radiador o del aceite del motor.

Mediante los circuitos que se describen a continuación, se puede disponer de instrumentos indicadores de la temperatura del agua del radiador o del aceite del 
motor. Para el primer caso, bastará poner el transductor en un lugar por el que pase una conducción de agua (radiador, culata, etc.); y para el segundo, en la parte inferior del bloque del motor (en el cárter, etc.).

Los transductores, en cualquiera de los dos casos, serán termistancias del tipo NTC con formas especialmente desarrolladas para su aplicación en automóviles, tal y como a continuación se muestra:

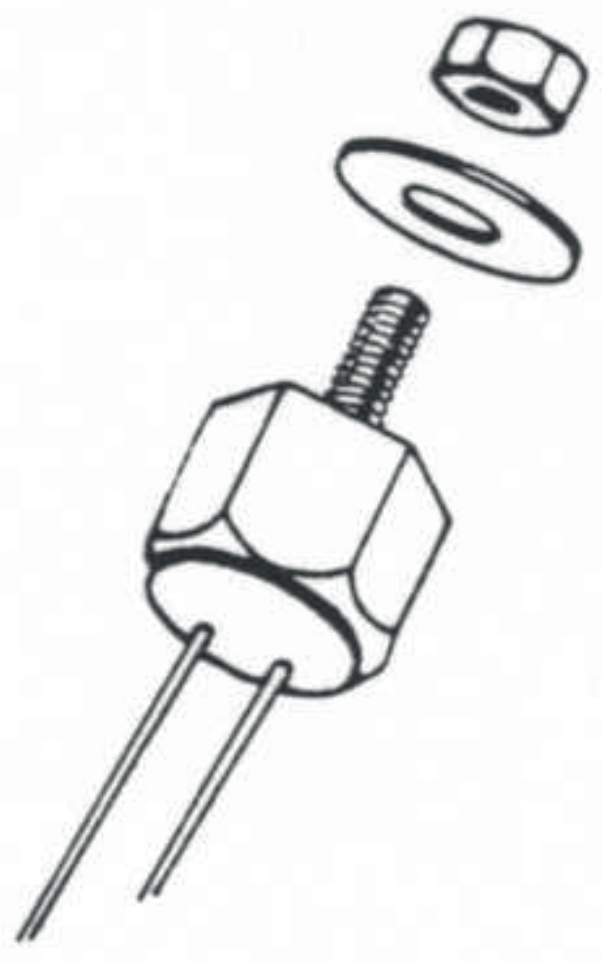

La diferencia entre los circuitos para la medida de temperatura de agua o de aceite será el tarado o calibrado del instrumento indicador, que, en el primer caso, se realizará para un mínimo de la escala de unos $40^{\circ} \mathrm{C}$ y un máximo de unos $100{ }^{\circ} \mathrm{C}$; y en el segundo, entre un mínimo de unos $60^{\circ} \mathrm{C}$ y un máximo de $140{ }^{\circ} \mathrm{C}$.

El primero de los circuitos que se presentan está constituido por una configuración en puente de Wheastone, donde R1, R3, R4 y la NTC forman el circuito típico de un puente de resistencias; a saber: 


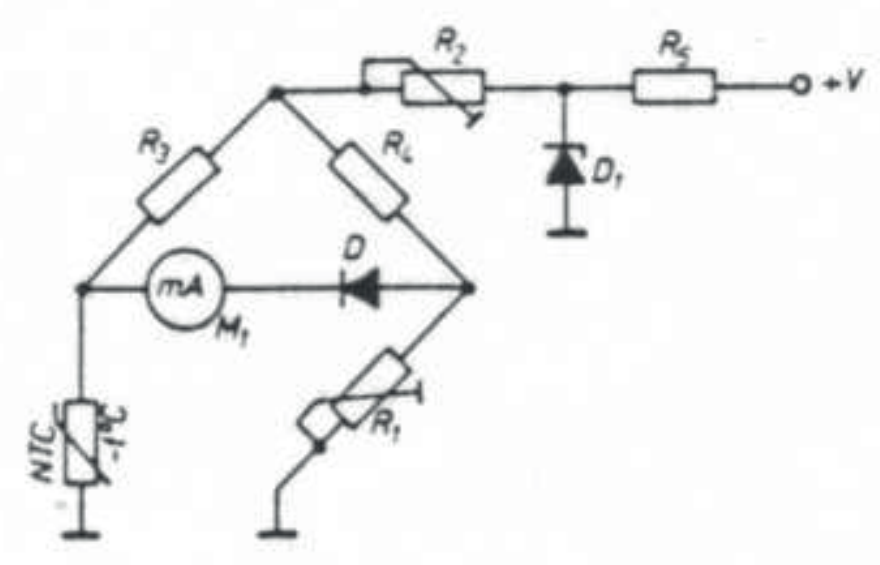

Para la temperatura máxima que se quiera medir, se ajusta R2 de manera que el instrumento indicador M1 (un miliamperímero) deflexione al fondo de la escala. R1 se ajusta para que a temperatura mínima no exista deflexión. Conviene repetir el ajuste de R1 y R2 por lo menos un par de veces con el fin de hacer el ajuste lo más fino posible. El diodo D se coloca para que, cuando la temperatura de la NTC sea menor que la mínima para la cual se taró, no circule corriente por el indicador M1 y su aguja permanezca en el límite inferior de la escala.

El funcionamiento del segundo circuito, se fundamenta en el empleo de un amplificador diferencial:

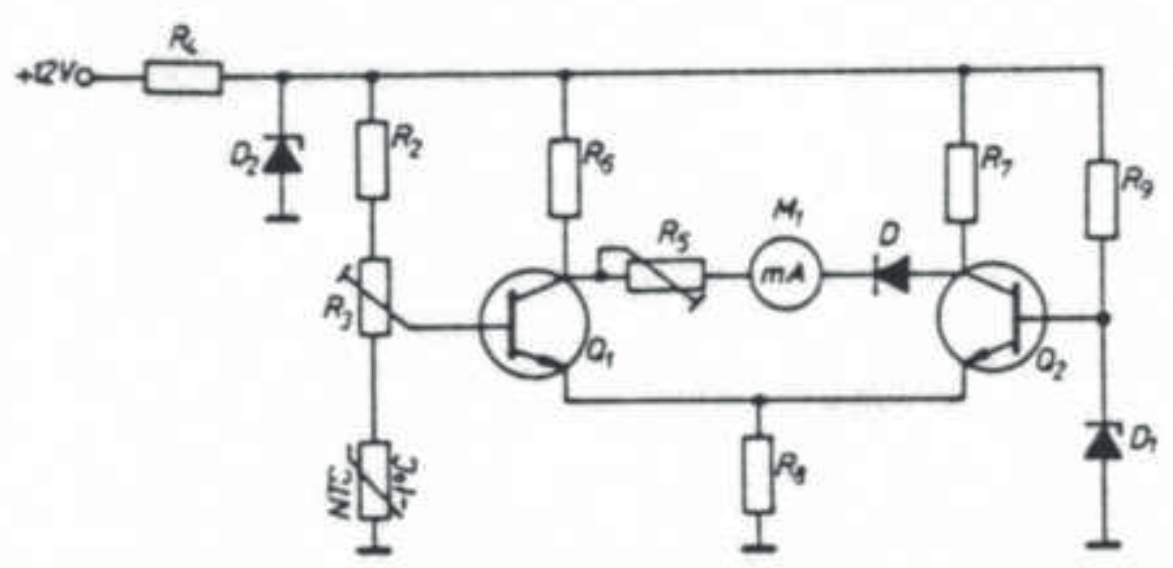


A una entrada de éste se le aplica una tensión fija proveniente de un estabilizador, que servirá como tensión de referencia (entrada al transistor Q2). A la otra entrada se le aplica una tensión variable y dependiente del valor de resistencia de la NTC (entrada al transistor Q1).

El tarado del instrumento se realiza por el procedimiento acostumbrado: R3 fija el mínimo de deflexión de la aguja de M1 para la temperatura mínima de la NTC, y R5 la máxima deflexión para la máxima temperatura. El diodo D juega el mismo papel que en el circuito anterior.

En estos circuitos conviene tener en cuenta dos observaciones:

La tensión de alimentación debe ser estabilizada para que el estado de carga de la batería no produzca informaciones de temperatura erróneas (por variaciones en su nivel de tensión).

El grabado en ${ }^{\circ} \mathrm{C}$ del instrumento indicador conviene hacerlo manualmente de cinco en cinco o de diez en diez grados y no interpolando entre la lectura máxima y mínima. Ello se hace con el objeto de corregir las alinealidades en la respuesta de la NTC.

Una variante de los circuitos indicadores de temperatura son los circuitos que proporcionan una señal acústica u óptica cuando la temperatura vigilada alcanza un determinado valor, superior al normal de funcionamiento. Estos circuitos están basados en circuitos disparadores Schmitt como el siguiente: 


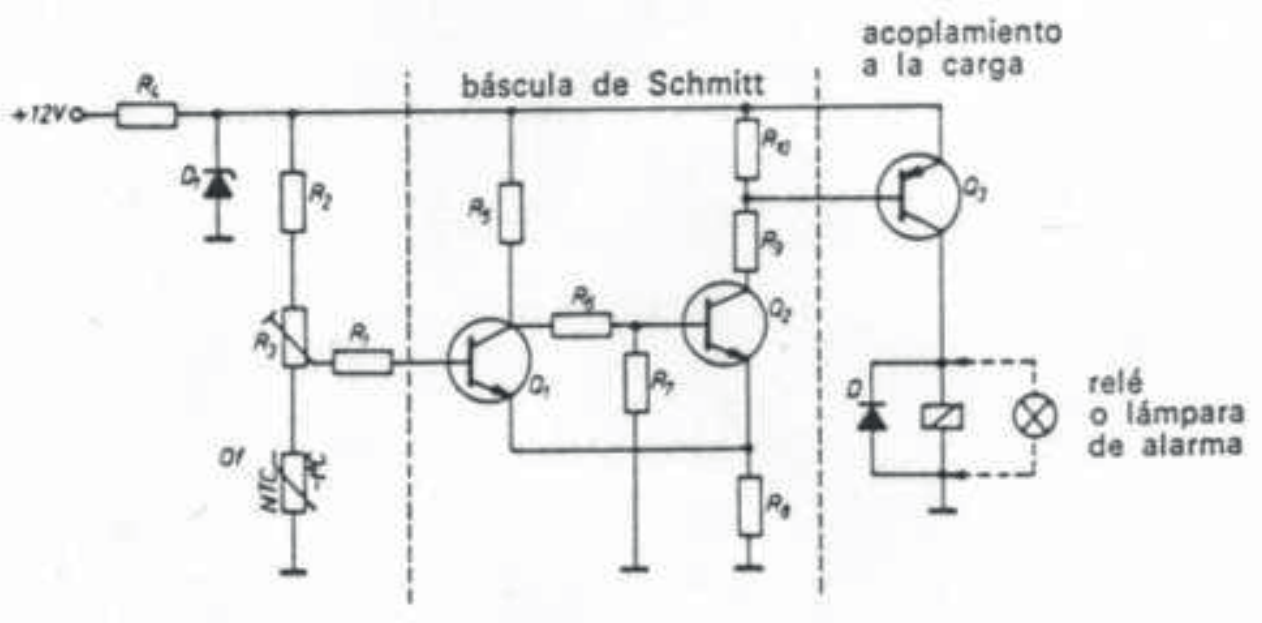

Los transistores Q1 y Q2 están montados sobre una configuración típica de báscula Schmitt, como se ha comentado. El transistor Q3 es un amplificador para acoplamiento de la carga (lámpara o relé) al circuito. El mecanismo completo para la excitación de la carga sería, tal y como se describe a continuación: Al aumentar la temperatura, la resistencia de la NTC disminuye hasta que la tensión de base de Q1 se hace tan pequeña que Q1 se bloquea (entra en corte) y automáticamente Q2 y Q3 pasan a saturación. El avisador acústico u óptico daría la alarma de sobretemperatura.

El ajuste de la temperatura, para la cual se debe disparar el circuito, se realiza por medio de la resistencia ajustable R3. Cuanto más hacia arriba esté el cursor, mayor deberá ser la temperatura para disparar el circuito.

Otros circuitos interesantes de ser comentados son aquellos que nos permitirán contabilizar el régimen de trabajo de nuestro motor. Los circuitos cuenta revoluciones para motores de explosión de gasolina suelen estar constituidos por un multivibrador monoestable, disparado por los impulsos de encendido y, cuya tensión de salida es leída en un instrumento indicador de bobina móvil o galvanómetro. En la siguiente figura se muestra uno de esos circuitos: 


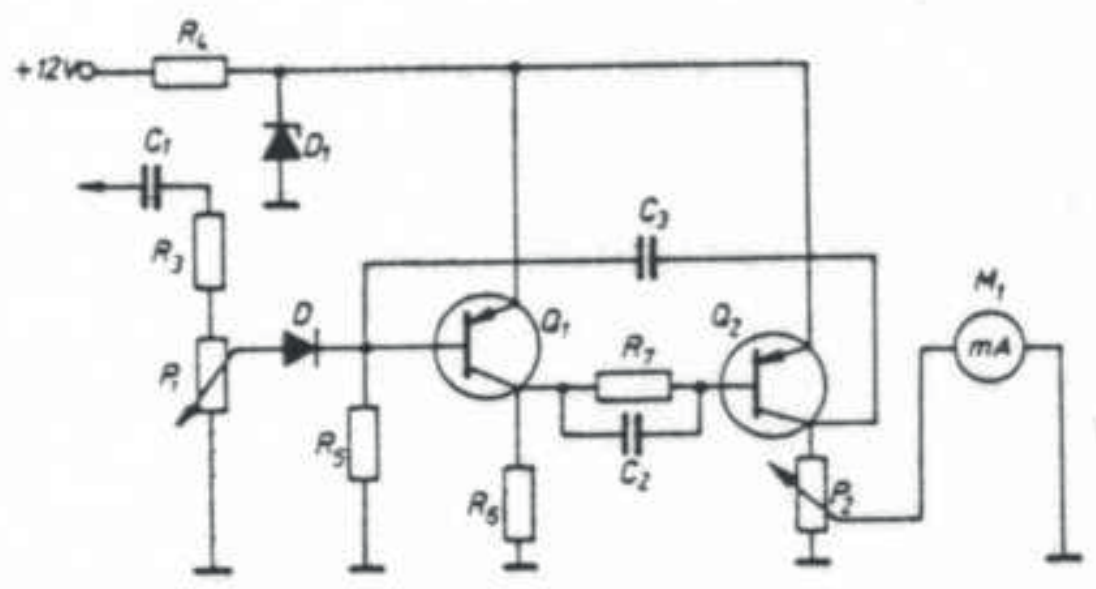

Los impulsos se toman de la bobina de encendido. Así, como es sabido, el número de impulsos de encendido del motor es directamente proporcional al número de revoluciones; el multivibrador monoestable se encargará de que todos los impulsos tengan una duración uniforme. El instrumento indicador, conectado en la carga del segundo transistor, apreciará los valores medios de la tensión existentes en esta resistencia, que serán proporcionales al número de impulsos producidos por la bobina. El potenciómetro P1 tiene como misión seleccionar una fracción de la amplitud del impulso, de forma que el monoestable sea excitado sin dificultad. El potenciómetro P2 fija el fondo escala del instrumento indicador para el máximo número de revoluciones del motor. El tarado de este instrumento debe hacerse con la ayuda de otro tacómetro, que servirá de referencia. La alimentación de este circuito también debe ser estabilizada convenientemente, para evitar los errores producidos por las diferentes cargas de la batería y con ello sus diferentes valores de tensión con el tiempo.

Actualmente existen en el mercado circuitos integrados especialmente desarrollados para su aplicación en circuitos cuenta - revoluciones de vehículos automóviles. Tal es el caso, por ejemplo, del integrado SAK 215, cuyo esquema de conexión se indica seguidamente: 


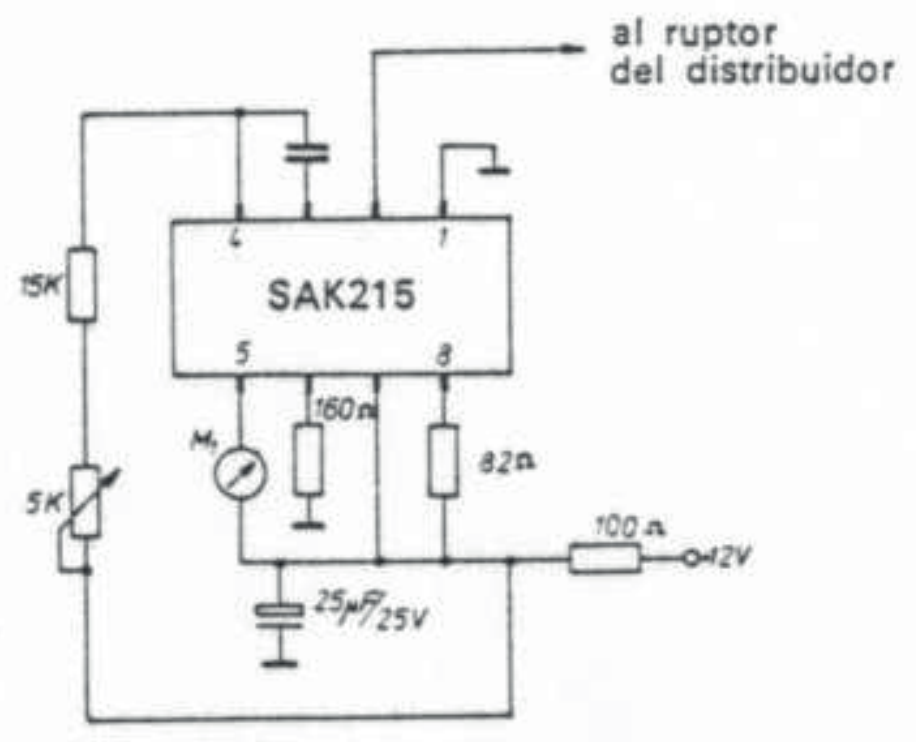

Tal como está el circuito, proporciona una indicación lineal sobre un miliamperímetro de $10 \mathrm{~mA}$ de fondo escala, para una deflexión máxima de 6000 vueltas en un motor que proporcione dos impulsos de ignición por cada vuelta de éste. Existen también en el mercado otros circuitos integrados especialmente desarrollados para su aplicación en el automóvil, tales como velocímetros electrónicos, avisadores de velocidad máxima, etc.; sus esquemas de aplicación son suministrados por las casas fabricantes en sus manuales de aplicación.

Otros circuitos electrónicos que me gustaría tratar son aquellos que gobiernan los indicadores de cambio de dirección. Suelen estar resueltos con disposiciones de circuitos que corresponden a multivibradores estables. Las diferencias entre unos y otros radican en la forma de acoplar la carga (lamparitas indicadoras) al circuito oscilador.

Atendiendo a estas diferencias, se pueden clasificar en circuitos que realizan la conexión y desconexión de las lamparitas mediante conmutadores electromecánicos 
(relés), y los que la realizan mediante conmutadores estáticos (tiristores). A continuación, se muestra un ejemplo de cada uno de ellos.

El circuito expuesto más abajo, corresponde a la primera categoría. Los transistores Q1 y Q2 forman una configuración típica de multivibrador estable, mientras que Q3 representa el acoplamiento entre la salida del multivibrador y el relé electromecánico. El multivibrador se proyecta de forma que oscile a razón de unos 40 ciclos por minuto.

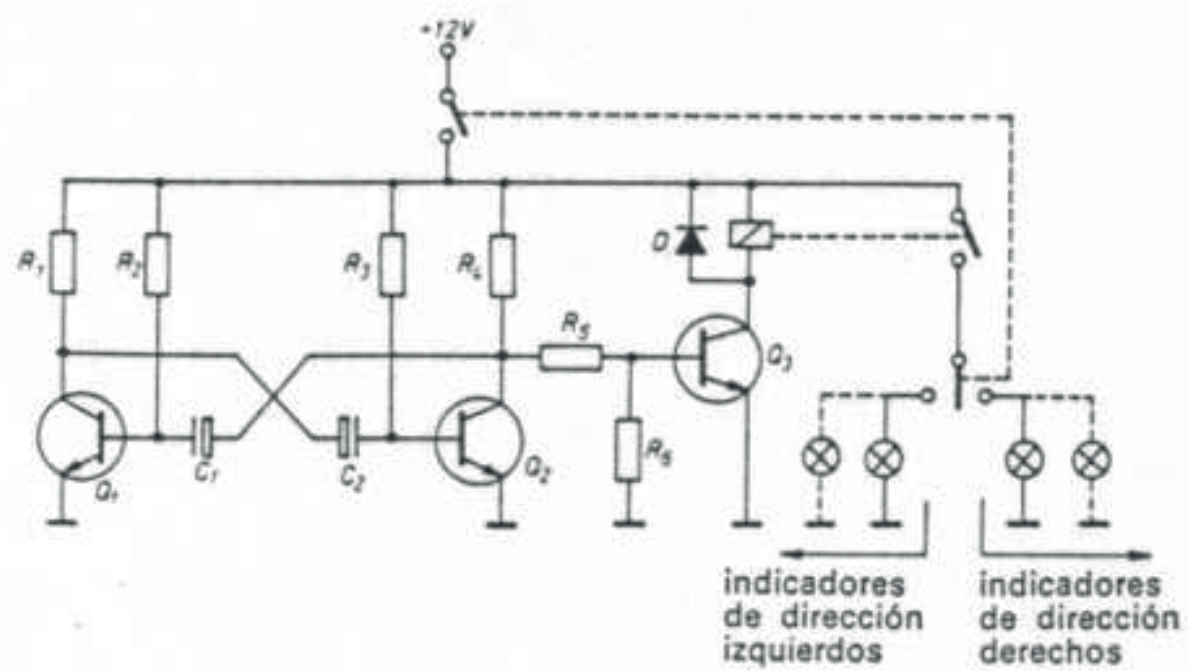

En este segundo, se representa un circuito mediante conmutadores estáticos. En sí, el circuito se comporta como un oscilador con tiristores: 


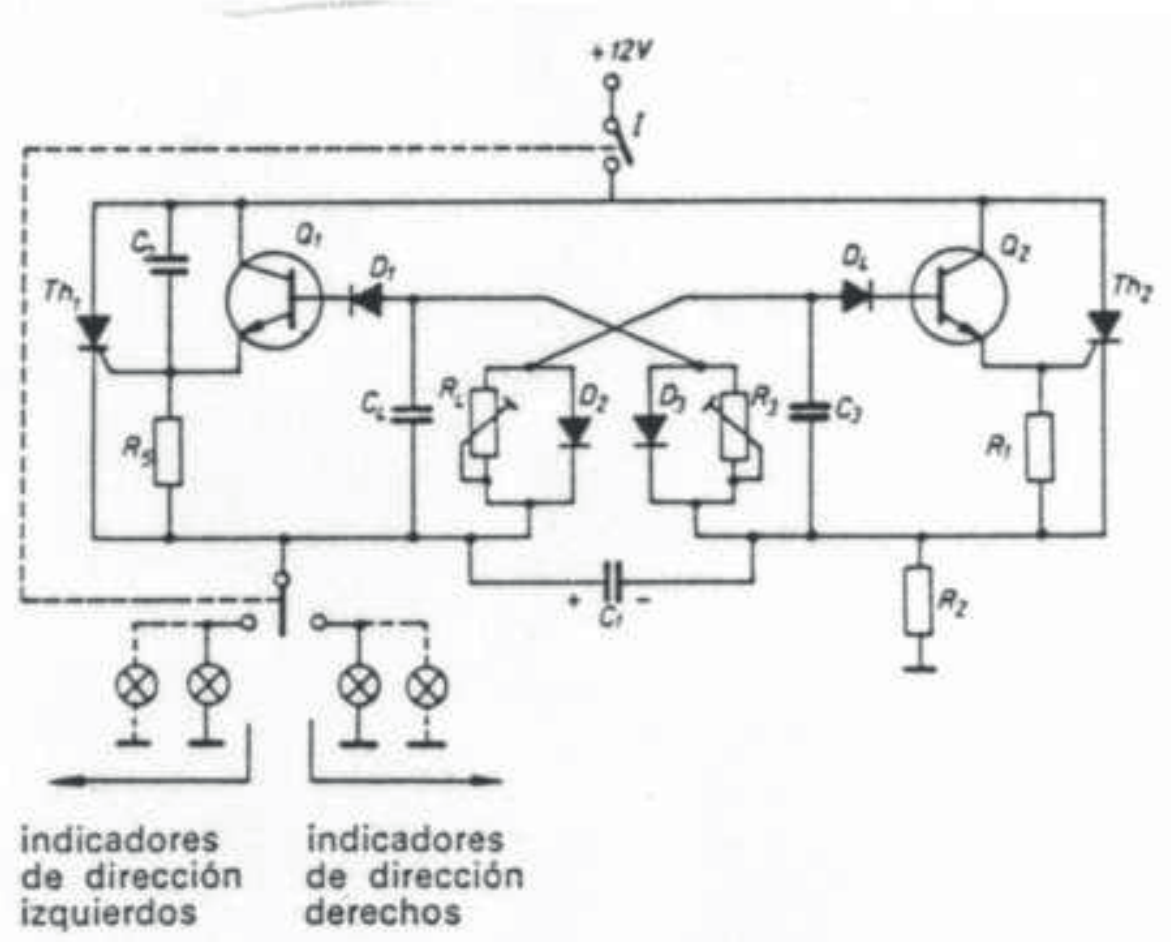

Cuando se cierra el interruptor, debido al hecho de que se quieren conectar los indicadores de la izquierda o de la derecha, disparará el tiristor Th1 y ello se debe a que, al querer cargarse $\mathrm{C} 2$, circula una corriente por R5, estableciéndose una diferencia de potencial entre sus extremos que hace que Th1 se cebe. Una vez cebado Th1, las lámparas seleccionadas se iluminan. En esta situación suceden los siguientes fenómenos en el circuito: el condensador C1 se carga aproximadamente a la tensión de alimentación con la polaridad indicada en la figura a través de $+12 \mathrm{~V}$, Th1 y R2; el condensador C3 tiende a cargarse también a la tensión de alimentación a través de + $12 \mathrm{~V}$, Th1, R4 y R2; la constante de carga de este condensador es mucho más elevada que la de $\mathrm{C} 1$ debido al valor relativamente elevado de R4. No obstante, C3 no llega a cargarse más de tres voltios, valor suficiente para que Q2 pase a saturación, aparezca tensión en R1 y Th2 se cebe. Cuando Th2 se ceba, el condensador C1 se coloca en paralelo con Th1, y por efecto de su carga, Th1 se bloquea. Al bloquearse Th1, las lámparas se apagan y la tensión de alimentación aparece en R2. Apenas Th2 se ha cebado, el diodo D2 comienza a descargar al condensador C3. 
En esta nueva situación, con Th2 cebado y Th1 bloqueado, se vuelven a repetir los mismos fenómenos anteriores: C1 se cargará a 12 voltios con polaridad contraria a la que se indica en la figura, C4 comenzará a cargarse a través de R3, hasta que adquiera el suficiente potencial como para conmutar a Q1; conmutado Q1, Th1 se cebará; C1 bloqueará a Th2, etc., etc.; los ciclos se repetirán, hasta que se corte la tensión de alimentación.

La frecuencia de oscilación de este circuito es regulable mediante R3 y R4, pudiéndose hacer incluso desiguales los tiempos de encendido $\mathrm{u}$ apagado de las lámparas.

Basado en el primero de los circuitos expuestos para las intermitencias, se podría también implementar el control de los limpiaparabrisas, sólo teniendo en cuenta dos pequeñas diferencias: que, en este caso, el multivibrador es asimétrico y, además, regulable. Mediante el potenciómetro P1, será posible ajustar la frecuencia del oscilador dentro de los límites de máxima y mínima cadencia de barrido.La única limitación de este montaje es que debe ser aplicado a motores de limpia - parabrisas dotados de contacto final de carrera para el retorno a la posición de reposo de las rasquetas limpiadoras.

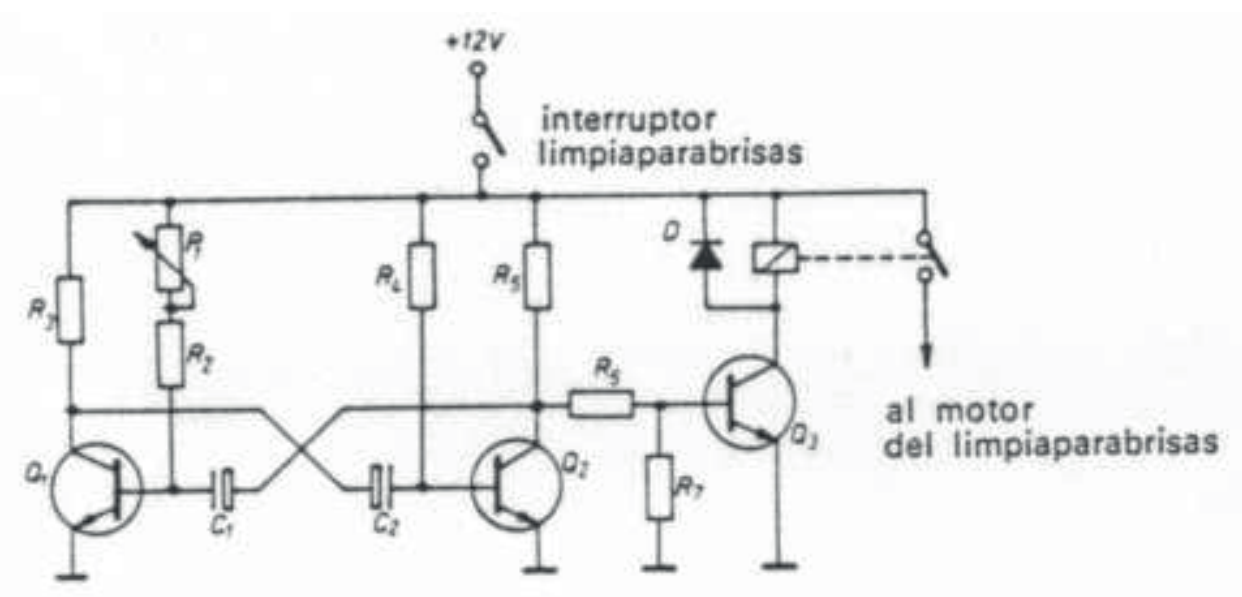


A continuación, describimos uno de los muchos circuitos que pueden ser utilizados como productores de tonos de avisadores acústicos en el interior de los vehículos, para diferentes tareas (puertas abiertas con el coche en movimiento, para avisar ciertas circunstancias, etc.). El circuito que a continuación se muestra es un poco más complejo que el simple multivibrador estable visto hasta ahora:

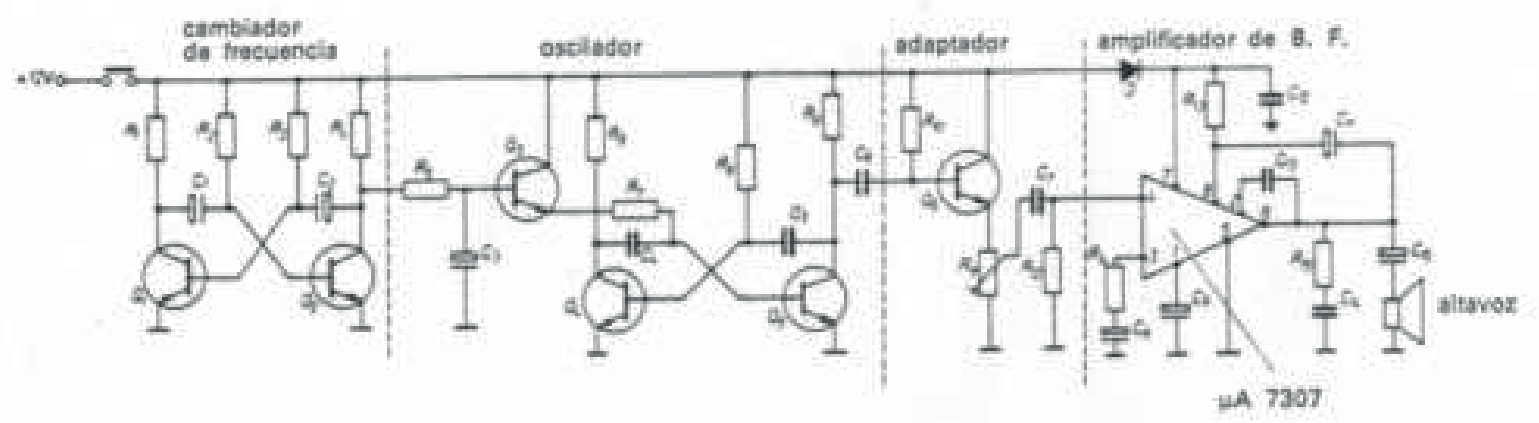

El circuito produce en el altavoz un sonido con una frecuencia continuamente variable entre unos 700 y $1400 \mathrm{~Hz}$, imitando las conocidas sirenas. El montaje puede dividirse en cuatro partes fundamentales: cambiador de frecuencia, oscilador, adaptador y amplificador de baja frecuencia. Tanto el cambiador de frecuencia como el oscilador son multivibradores estables. El del cambiador es de una frecuencia muy baja, entre 1 y 0,1 Hertzios, siendo la frecuencia del oscilador de $700 \mathrm{~Hz}$ para la máxima tensión colector - emisor de Q3 (en realidad controlada por el valor de la resistencia equivalente colector - emisor de este transistor), que, a su vez, es controlado por la señal cuadrada de salida, convenientemente integrada por R5 y C3, del multivibrador estable cambiador de frecuencia. Se integra la salida del primer circuito para que la transición de frecuencia del oscilador no sea brusca (conmutación con onda cuadrada), sino paulatina.

La señal de salida del oscilador modulada en frecuencia es llevada a través de C6 a la etapa adaptadora formada por Q6 y, de ésta, a un amplificador de B. F. que, en este circuito, se ha puesto con un circuito integrado (concretamente el m A 7307), pero que igualmente podría ser otro amplificador con componentes discretos. 
Una aplicación interesante del circuito anteriormente descrito es la del avisador acústico de fallos del motor, como por ejemplo: falta de presión de aceite, temperatura excesiva del agua del radiador, etc.

Aquí termina la presente entrega sobre electrónica básica aplicada al automóvil. Les espero en la próxima. Si alguien pudiera aportar sus conocimientos, para expandir los aquí expuestos con nueva información, o con el fin de rebatir errores que yo haya cometido en esta exposición, o manifestar críticas (a ser posible constructivas por favor), o cualquier tipo de comentario, les animo a que se dirijan a esta magna revista para que aquí me "abronquen" convenientemente por "manta" (hago aquí la solemne promesa de que, en cuanto lo sepa, ahora que manejo algo mejor, no mucho por cierto, este diabólico artefacto de los "internés de la güeb", publicaré, si ello fuere menester, claro, aquello que se me enviare).

Espero que haya resultado del gusto del lector. Si no fue así, desde aquí hago propósito de enmienda, para que el próximo artículo salga mejor (eso si no me echan antes por "rollista"). Si, por el contrario, le gustó, y despidiéndome una vez más al más puro estilo Shakesperiano, (llegará a convertirse en una tradición esta forma de despedirse y sino al tiempo) quedemos como amigos y volvamos a encontrarnos donde a la diosa fortuna más la complazca. 